Cinémas

Revue d'études cinématographiques

Revue d'études cinématographiques

Journal of Film Studies

\title{
Hollywood, Rossellini et le syndrome de Griffith
}

\section{Elena Dagrada}

Volume 13, numéro 1-2, automne 2002

Limite(s) du montage

URI : https://id.erudit.org/iderudit/007959ar

DOI : https://doi.org/10.7202/007959ar

Aller au sommaire du numéro

Éditeur(s)

Cinémas

ISSN

1181-6945 (imprimé)

1705-6500 (numérique)

Découvrir la revue

Citer cet article

Dagrada, E. (2002). Hollywood, Rossellini et le syndrome de Griffith. Cinémas / Revue d'études cinématographiques, 13(1-2), 129-141.

https://doi.org/10.7202/007959ar
Résumé de l'article

Les films que Rossellini a tournés dans les années 1950 avec Ingrid Bergman existent tous en plusieurs versions. Il existe notamment trois versions de Stromboli, dont l'une qui fut montée aux États-Unis par la RKO. L'étude des différences entre la version américaine et les deux autres versions nous en dit beaucoup sur l'approche du montage propre à Rossellini, ainsi que sur l'approche du montage propre à Hollywood, affectée par ce que l'on propose ici d'appeler le " syndrome de Griffith » et fondée sur une prédilection envers le morcellement de l'espace et le recours au montage alterné. On verra ici que le montage de la RKO conduit le film de Rossellini à une dimension mélodramatique totalement étrangère à la sensibilité du réalisateur italien. 


\section{Hollywood, Rossellini et le syndrome de Griffith}

\section{Elena Dagrada}

\section{RÉSUMÉ}

Les films que Rossellini a tournés dans les années 1950 avec Ingrid Bergman existent tous en plusieurs versions. Il existe notamment trois versions de Stromboli, dont l'une qui fut montée aux États-Unis par la RKO. Létude des différences entre la version américaine et les deux autres versions nous en dit beaucoup sur l'approche du montage propre à Rossellini, ainsi que sur l'approche du montage propre à Hollywood, affectée par ce que l'on propose ici d'appeler le «syndrome de Griffith" et fondée sur une prédilection envers le morcellement de l'espace et le recours au montage alterné. On verra ici que le montage de la RKO conduit le film de Rossellini à une dimension mélodramatique totalement étrangère à la sensibilité du réalisateur italien.

\section{ABSTRACT}

Several versions exist of all the films that Rossellini made during the 1950s with Ingrid Bergman. There are in fact three versions of Stromboli, one of which was edited in the United States by RKO. Studying the differences between the American version and the other two versions sheds light on the editing approach used by Rossellini and the editing approach used by Hollywood. The latter was affected by what we propose to call the "Griffith Syndrome" and it is based on a predilection for the fragmentation of space and the use of cross-cutting. We will observe how RKO's editing steers Rossellini's film into a melodramatic dimension, totally foreign to the mindset of the Italian director. 
Dans un document anonyme consacré aux coupes et aux remontages subis par le film Stromboli, premier film de Roberto Rossellini interprété par Ingrid Bergman, les coupes opérées sur la version américaine du film sont commentés sur un ton résolument polémique. Il s'agit d'un document sans doute postérieur aux années 1950, probablement écrit par Rossellini lui-même lorsqu'il présidait le Centro Sperimentale di Cinematografia, entre 1969 et $1974{ }^{1}$. En tous les cas, qu'il s'agisse ou non de Rossellini, l'auteur paraît parfaitement conscient que la singularité de la version américaine ne tient pas seulement à la suppression de tel ou tel plan ou de telle ou telle scène, mais procède d'un changement radical dans le style du film, suite à son remontage.

On sait qu'il existe au moins trois versions officielles du film Stromboli ${ }^{2}$. La première, anglophone, est distribuée aux ÉtatsUnis par la RKO à partir du 15 février 1950. La seconde, destinée au marché international et anglophone elle aussi, est d'abord présentée à la presse romaine au cours du mois de mars 1950 ; puis, elle est projetée hors compétition à la XI Mostra d'arte cinematografica de Venise le 26 août suivant (le même jour que Francesco giullare di Dio), et sort à Paris le 20 octobre de la même année, et ce sans jamais avoir été distribuée dans les salles italiennes. Enfin, une troisième version (intitulée Stromboli terra di Dio), doublée en italien et amputée d'à peu près six minutes par rapport à la version internationale, sort sur les écrans de Turin à partir du 8 octobre 1950.

Les raisons pour lesquelles ces différentes versions existent sont nombreuses et fort complexes, mais résident entre autres dans le fait que Rossellini se soit méfié de la RKO et de son patron, Howard Hughes. En effet, sa défiance était telle qu'il tourna pratiquement ce film deux fois, ayant pris soin d'obtenir deux négatifs distincts ${ }^{3}$. Stromboli était produit par Hughes pour le compte de la RKO, mais aussi par Rossellini et Bergman pour celui de la Berit (acronyme qui dérive des initiales de Bergman, Rossellini et Italia). Rossellini était tenu par contrat d'envoyer un négatif aux États-Unis, mais en tournant deux fois chaque plan, y compris les plans raccords, il se donnait la chance d'exécuter "son" montage en Italie et d'envoyer aux 
États-Unis une version du film comme modèle à suivre, lorsque celui-ci serait monté par la $\mathrm{RKO}$.

Malgré ces précautions, la version américaine du film ne correspondit pas aux attentes de Rossellini : il refusa donc de la reconnaître - même s'il en avait tourné chaque plan — et intenta une action contre la maison de production hollywoodienne $^{4}$, coupable à ses yeux d'avoir distribué un film dont le montage était infidèle ${ }^{5}$.

Si l'on compare aujourd'hui, plan par plan, ces trois versions de Stromboli, on s'aperçoit à quel point elles reflètent deux conceptions du montage diamétralement opposées. On a, d'une part, ce qu'on pourrait appeler l' "anomalie» rossellinienne qui, par son refus du morcellement de l'espace ainsi que du montage invisible et de ses conventions les plus apparemment "universelles", témoigne d'un vrai cas limite, à l'époque, de modernité cinématographique; d'autre part s'affirme une vision hollywoodienne du montage, liée au modèle de la segmentation analytique de l'espace et de l'alternance tel qu'il a été mis au point surtout par David W. Griffith, modèle à partir duquel on a voulu effacer l'empreinte du réalisateur italien de la version américaine de son film.

Selon les intentions de Rossellini, le final cut de la version américaine devait correspondre à une copie montée par lui, en collaboration avec Jolanda Benvenuti, et expédiée aux États-Unis en début de décembre $1949^{6}$. Ceci est attesté par Ingrid Bergman, dans une lettre écrite le 6 décembre et adressée à sa belle-sœur Anita, qui à l'époque était aux États-Unis où son mari Renzo Rossellini, musicien réputé, devait veiller à la mise au point du film et travailler à sa musique ${ }^{7}$. Dans cette lettre, Bergman annonce l'arrivée imminente de cette "final cut version", mais ne cache pas sa crainte que quelqu'un puisse vouloir la remanier ${ }^{8}$. Ce qui ne manqua pas de se produire: dès son arrivée à destination, le film fut confié par Howard Hughes aux soins du monteur Roland Gross; il subit donc quelques modifications avant d'être l'objet d'une projection privée à Santa Monica, où un public composé d'acteurs et de techniciens réagit négativement ${ }^{9}$. Hughes décida alors d'abréger et de modifier le film à nouveau, confiant son remontage à Alfred Werker, non crédité au générique. 
Distribuée aux États-Unis peu de temps après, cette version est très différente des versions internationale et italienne distribuées par la suite en Europe, et ce pas seulement à cause des différentes prises de vue qui la composent.

En apparence, l'intrigue demeure la même: la «grande et blonde" Karin (c'est en ces mots que Rossellini décrit le personnage, et ce dès la première version du scénario ${ }^{10}$ ), expatriée lituanienne, épouse un pêcheur sicilien nommé Antonio et le suit dans son village afin d'échapper au camp de Farfa Sabina, situé aux alentours de Rome, où elle est enfermée après la guerre. Très tôt, Karin s'aperçoit qu'elle a quitté une prison pour une autre: une île menacée par un volcan, dont les habitants sont hostiles et sauvages. Enceinte, Karin cherche alors à s'échapper du village avec l'aide de Cola, le gardien du phare, puis gagne les pentes du volcan. Dans la version américaine, après avoir passé une nuit au sommet du volcan, Karin retourne au village et accepte la vie qui l'attend auprès de son mari; dans les deux autres versions, elle reste au sommet du volcan et demande à Dieu de l'aider.

Cependant, en remaniant le film comme elle l'a fait, la RKO parvient à lui donner une dimension mélodramatique absente des deux autres versions proposées par Rossellini, en accentuant par le montage l'opposition culturelle entre la "grande et blonde" protagoniste du film et son «noir» environnement naturel (le volcan) ainsi que social (les habitants "hostiles»). Dans ses versions du film, Rossellini insiste au contraire davantage sur la dimension spirituelle du conflit (dimension du conflit que la RKO cherche à supprimer en toute occasion), en opposant la protagoniste à Dieu, que la nature est censée personnifier ${ }^{11}$.

Ce qu'on note d'abord, dans cette version dénaturée par la RKO, c'est la présence d'une voix off, impersonnelle et aseptisée, qui a pour but d'introduire le public américain au contexte géographique et culturel du récit, ainsi que de rendre la narration plus fluide.

On note aussi que cette version contient un certain nombre de plans que Rossellini a tournés mais supprimés des versions internationale et italienne et qui à eux seuls peuvent susciter l'intérêt de tout chercheur intéressé par l'œuvre rossellinienne. 
Le cas le plus flagrant est sans doute celui d'une scène composée de trois plans assez longs ${ }^{12}$, où Antonio, au lendemain d'une violente dispute avec Karin, l'oblige à l'accompagner dans le cimetière de l'île: il est vraisemblable que la RKO ait conservé cette scène en raison du fort conflit culturel qu'elle exprime, opposant l'homme méridional profondément attaché a des valeurs religieuses traditionnelles et la femme "du Nord», insensible à ce genre de croyances.

Mais nombreuses sont les autres modifications du genre à avoir été apportées au film de Rossellini. Signalons, par exemple, le début et la fin du film: dans la version RKO, grâce au recours conjoint à la voix off et à un nouveau montage, le style et le sens même de ces scènes sont altérés, car le film commence par une présentation de l'île et de ses habitants, ce qui n'est pas le cas dans les deux autres versions, et surtout s'achève par le retour de Karin au village, sans que celle-ci n'ait

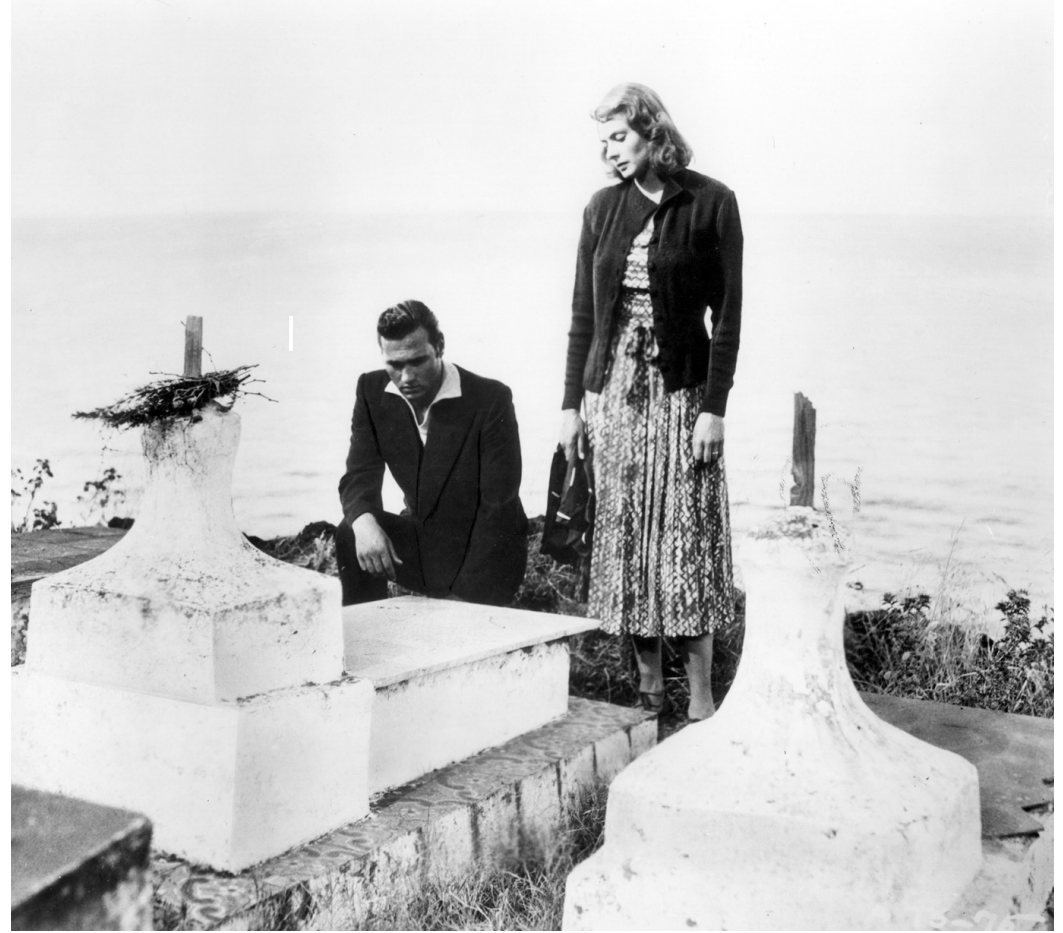

Stromboli, Roberto Rossellini, 1950 (Collection Adriano Aprà) 
invoqué Dieu. Ce retour de Karin au village est commenté en voix off et suggéré par un montage refait ad hoc à partir de trois plans inutilisés dans les autres versions (un premier où Karin se nettoie le visage avec son foulard; un second où elle descend du volcan; un troisième où elle sourit en gros plan) et d'un plan final représentant le village en profondeur de champ — village qui semble se rapprocher au fur et à mesure que progresse un «faux travelling in truca» qui simule un mouvement vers l'avant.

Notons également que de nombreux plans tournés par Rossellini ne sont pas utilisés dans la version RKO. Au total, cette version est en effet beaucoup plus courte que les autres: elle dure près de vingt minutes de moins que la version internationale et à peu près une demi-heure de moins que la version italienne. Presque toutes les séquences ont été abrégées afin d'accélérer le rythme du film et d'en éliminer les prétendus temps morts - les célèbres et magnifiques temps morts de l'attente rossellinienne. Les séquences de la pêche au thon, de la déambulation de Karin dans le labyrinthe que composent les ruelles du village, du mariage, du voyage vers l'île de Stromboli, entre autres, sont amputées.

Cependant, ce qui différencie vraiment la version RKO des deux autres est le recours à un nouveau style de montage, comme l'auteur du document anonyme cité au début de cet article l'avait bien compris. Un nouveau style de montage qui résulte à la fois de la multiplication des plans et de la réduction systématique de leur durée moyenne. Ce qui domine, ici, c'est une pratique de recyclage des plans (un «recyclage endogène», selon la terminologie proposée par Nicole Brenez ${ }^{13}$ ), on pourrait même dire leur remploi systématique - que ce soit par leur segmentation, leur dédoublement ou leur redistribution chronologique. Qu'il s'agisse de simples plans raccords ou de plans plus longs et plus articulés, ils sont continuellement réutilisés, morcelés, redistribués, s'acquittant de différentes fonctions, utilisant un geste isolé pour bâtir une succession de plans en montage alterné, ou transformant une longue prise de vues en une articulation de champs-contrechamps.

Ces interventions ne sont jamais fortuites: elles paraissent être dans la dépendance d'une conception proprement holly- 
woodienne du montage. Une conception fort différente de celle de Rossellini, qui interrompt le moins possible par le biais du montage ce qui peut être montré en continu à l'écran. Au contraire, la logique du montage hollywoodien consiste en l'analyse et en la fragmentation de l'espace. C'est cette logique, mise au point aux États-Unis dès les années 1910, qui prédomine dans la version américaine de Stromboli, où les interventions les plus fréquemment apportées par la $\mathrm{RKO}$ renvoient à ce que nous pourrions appeler le "syndrome de Griffith", voire à la segmentation de l'espace par le recours au montage alterné ${ }^{14}$.

Rossellini évite autant que possible ces pratiques de segmentation, particulièrement à compter de sa collaboration avec Ingrid Bergman; la RKO le «corrige» donc, le bride à sa façon, modifiant le montage du film en utilisant un plus grand nombre de plans, en réduisant leur durée et en les alternant chaque fois que cela paraissait nécessaire.

Citons seulement quatre occasions où la RKO a jugé bon de "corriger" le montage de Rossellini, même si bien d'autres exemples sont envisageables. Le plus éloquent de ces cas, d'ailleurs, est sans doute celui évoqué plus haut, où la scène composant la fin du film est remaniée par un montage alterné. Ce cas est exemplaire parce que la descente de Karin du volcan vers le village y est tout simplement suggérée par l'insertion de trois plans déjà décrits (celui où Karin se nettoie le visage avec son foulard; celui où Karin descend du volcan; enfin, celui où Karin sourit en gros plan), montés en alternance avec un plan du village en profondeur de champ, utilisé trois fois et manipulé à son tour, en dernier lieu, par la simulation d'un mouvement vers l'avant.

Un second exemple, tout aussi éloquent, nous est fourni par une scène se déroulant à son tour vers la fin du film, où Antonio séquestre Karin dans leur maison. Dans les versions internationale et italienne, un seul plan extérieur est utilisé - plan qui représente Antonio clouant la porte d'entrée - tandis que le reste de cette action est composé de plans intérieurs, montrant les réactions de Karin; dans la version RKO, les plans d'Antonio clouant la porte sont au nombre de trois et sont insérés de 
manière à alterner avec les plans où Karin est à l'intérieur de la maison, selon une logique de montage alterné.

Troisième exemple: le traitement que réserve la RKO à la scène où Karin et Cola, le gardien du phare, se rencontrent pour la première fois, sur un bateau se dirigeant vers l'île de Stromboli. Tandis que dans les versions internationale et italienne, Cola observe Karin dormant aux côtés de son mari sans qu'intervienne de jeu de regards (jusqu’à un fondu enchaîné qui interrompt la première partie de cette scène), la version américaine utilise des plans délaissés par Rossellini qui, montés en alternance, décrivent un jeu de regards entre Cola et Karin : celle-ci, consciente d'avoir séduit le gardien du phare, sourit d'un air cynique.

Dans un traitement du sujet que Rossellini avait développé lorsqu'il se trouvait aux États-Unis en 1949, le thème du triangle amoureux — thème propice au mélodrame s'il en fut — est effectivement amorcé et c'est ce thème que la RKO développe à son tour, comme on vient de le voir, au moyen du montage. Le montage est par ailleurs utilisé par la RKO d'une manière semblable pour rendre équivoque la relation entre Karin et un autre personnage: le curé du village. Karin et le curé se croisent souvent dans le film, et dialoguent en tête à tête à deux reprises. Les remontages que la RKO propose de ces dialogues sont très intéressants et nous fournissent donc un quatrième exemple.

Le premier échange entre Karin et le curé a lieu au bord de la mer. Tandis que dans les deux autres versions le curé s'y rend accompagné d'un jeune garçon, dans la version $\mathrm{RKO}$ il s'y rend seul, ce qui donne à sa rencontre avec Karin un caractère vaguement clandestin. Là encore prime la construction en montage alterné: un plan représentant Karin, inutilisé dans les autres versions, est ici monté en alternance avec un plan montrant le curé s'approchant d'elle. Le deuxième échange a lieu au presbytère. Cette séquence, telle qu'elle est montée par Rossellini, s'amorce abruptement par un plan rapproché de Karin qui regarde devant elle (presque en direction de la caméra), s'adressant à un inconnu; l'inconnu est graduellement découvert par la caméra opérant un lent mouvement vers l'arrière, tandis que Karin avance dans sa direction. Dans la version RKO, la 
séquence s'ouvre par un plan d'ensemble de l'église — plan, là encore, inutilisé dans les autres versions - afin d'introduire de manière conformément hollywoodienne le lieu où va se dérouler l'action; ce plan d'ensemble est suivi d'un plan rapproché de Karin, puis d'un gros plan du curé en contrechamp. Or, ce gros plan, Rossellini ne l'utilise pas de cette façon: il s'agit en fait d'un fragment d'un plan du curé, beaucoup plus long et composite (situé par ailleurs beaucoup plus tard dans la séquence des versions internationale et italienne), anticipé ici par la RKO afin de "normaliser» un début de séquence aussi contraire à la norme par un rassurant champ-contrechamp.

En somme, c'est une véritable lutte que se livrent Rossellini et la RKO : le montage en est l'arme et le style l'enjeu. La RKO ne souffre pas les prises de vues continues de Rossellini, et cherche à l'«hollywoodiser» en reconstruisant son film selon les paramètres du langage cinématographique classique et du montage analytique, notamment afin que l'intrigue atteigne un niveau suffisamment mélodramatique. De son côté, Rossellini montre bien - dans les versions internationale et italienne de Stromboli - à quel point il refuse d'envisager le montage comme outil de segmentation (et de manipulation) de l'espace-temps et demeure réfractaire à toute contamination rhétorique.

En effet, la logique analytique du montage alterné, tel que le pratique la RKO, apparaît souvent contaminée par une logique associative d'ordre rhétorique. Par cette logique associative, un lien complexe et puissant - que Rossellini ne propose pas est souvent établi entre des personnages et leurs environnements, ou encore entre des paysages et les événements qui s’y déroulent. L'alternance entre intérieur et extérieur, par exemple, ou entre champ et contrechamp, ne se limite pas nécessairement à représenter deux séries simultanées en tant que telles, mais entraîne parfois leur association métaphorique, symbolique ou comparative, selon le principe de cet autre procédé griffithien par excellence qu'est le montage parallèle ${ }^{15}$.

C'est d'ailleurs bien souvent dans le but de produire ce genre d'associations métaphoriques que la RKO segmente des plans et les multiplie, selon le procédé que nous avons décrit plus haut. Par exemple, on multiplie des plans représentant le paysage 
volcanique, le panorama du village ou la mer poissonneuse, puis on les exploite à des fins métaphoriques en les alternant avec des plans dramatiques, afin de suggérer que la nature participe aux événements narrés, conformément aux codes du mélodrame. Il est d'ailleurs fréquent que ces plans multipliés soient insérés dans le récit en guise de glose mélodramatique que l'on accompagne d'une musique redondante - musique dans l'ensemble plus présente ici que dans les versions internationale et italienne. Que les images soient celles du menaçant volcan ou de l'île encerclée par la mer, systématiquement, on les agence de sorte qu'elles produisent des métaphores tissant un lien entre la nature et le drame vécu par les protagonistes, ou qu'elles commentent de façon encore plus spectaculaire les épisodes les plus dramatiques.

On trouve un exemple intéressant de ce procédé à la fin d'une séquence où Antonio et Karin se disputent. Dans la version $\mathrm{RKO}$, deux plans sont insérés entre cette séquence et la suivante, l'un proposant une vue nocturne et l'autre une vue diurne du village, éclairé par la lumière de l'aube, les deux plans étant liés par un fondu enchaîné: ceci afin de souligner le passage de la nuit au jour, certes, mais aussi afin de suggérer qu'il existe un lien entre la dispute du couple et le cycle des jours. Autre exemple: Karin est chez elle dans la cuisine lorsque le volcan entre en éruption; dans la version RKO, entre un plan montrant la première explosion du volcan et un autre où Karin paraît effrayée dans sa cuisine, on intercale un plan du cratère fumant - utilisé par Rossellini à un autre moment du film - , suggérant ainsi de façon redondante que la vie de la protagoniste est menacée. Troisième exemple: vers la fin du film, Karin monte au sommet du volcan; dans la version RKO, on intercale entre des plans illustrant son cheminement au travers des broussailles deux plans du paysage plongé dans une dense fumée — images qui se veulent prémonitoires de sa défaite —, en suivant encore une fois le modèle analytique du montage alterné, utilisé ici pour créer des significations symboliques.

Mais l'exemple peut-être le plus significatif nous est fourni par une séquence en début de film, qui s’amorce par l'arrivée de Karin à sa nouvelle demeure - petit bâtiment délabré, très 
différent du paradis méditerranéen que la femme avait imaginé - et se conclut par un mouvement panoramique de la caméra qui quitte Karin, incrédule et effarée, pour cadrer le volcan. Dans la version $\mathrm{RKO}$, on récupère ici un plan moyen du cratère fumant qu'on insère entre les autres, ceci afin d'insister sur le fait que la vie de la jeune femme est menacée et d'encourager la participation émotive du spectateur même le plus distrait.

C'est bien cette séquence qui, entre autres, est commentée en détail dans le document anonyme évoqué au début de cette étude, où le rapprochement incongru et prématuré entre Karin et le volcan est critiqué, car de cette manière le «mystérieux personnage est ainsi dévoilé dès le tout début, jusque dans ses tripes ${ }^{16}$ !» C'est en effet ce type de montage - ou plutôt de remontage - qui parvient à produire un changement de style dont les conséquences vont bien au-delà de celles qu'entraîne une simple opération de coupe et de suppression de plans ou de séquences. Car il s'agit d'un type de montage qui vise non seulement à apprivoiser le langage cinématographique, mais également à rendre le récit plus fluide, plus clair et plus conséquent, c'est-à-dire selon le goût d'Hollywood, où l'on aime que tout soit bien expliqué. Surtout, ce type de montage vise à enfermer le film dans les limites du mélodrame: redondance rhétorique des images; alternance de plans visant à créer du suspense ou des métaphores symboliques (souvent spectaculaires); gloses du récit conditionnées par un imaginaire romantique de la nature - imaginaire totalement étranger à la sensibilité de Rossellini qui, au contraire, met en scène la nature dans une perspective mystique et sensuelle.

D'emblée - dès que fut choisi le premier titre annoncé à la presse (After the Storm), lorsque Rossellini était aux États-Unis à la recherche d'un financement pour réaliser son film -, Hollywood avait l'intention de produire un mélodrame. On voulait un mélodrame sentimental, nébuleux, riche en sous-entendus: c'est vraisemblablement pourquoi, une fois qu'elle eut pris possession des images de Rossellini, la RKO a gardé la scène du cimetière, souligné la possibilité d'une liaison amoureuse entre Karin et Cola ainsi que l'ambiguïté du lien unissant Karin et le curé, éléments mélodramatiques que Rossellini, au contraire, s'est efforcé d'estomper. 
Fidèle à son style, le réalisateur italien voulait de son côté réaliser un film libre de toute contrainte générique et de toute convention langagière. En ne respectant pas cette volonté, la maison de production RKO donna de bonnes raisons à Rossellini d'intenter une action en justice contre elle, de même que le public américain avait de bonnes raisons de ne pas apprécier cette version du film - dont les recettes relativement considérables sont imputables au scandale que suscita Ingrid Bergman en abandonnant Hollywood, en divorçant de son mari suédois et en ayant un enfant de Rossellini hors mariage. La version RKO du film est en effet un objet cinématographique hybride qui ne parvient ni à apprivoiser la force rebelle des images rosselliniennes, ni à être aussi intensément suggestif qu'un authentique mélodrame hollywoodien.

C'est peut-être cette expérience avec la RKO qui, par la suite, a conduit Rossellini à épurer son style toujours davantage, jusqu’à ne presque plus tourner de plans raccords - si faciles à recycler et à multiplier - afin de garantir ses films contre des montages apocryphes. Après Stromboli, bien sûr, d'autres tenteront de manipuler ses films, en intervenant entre autres sur le montage, mais il sera de plus en plus difficile de le faire.

Università degli Studi di Milano

\section{NOTES}

1. Cet article a été écrit dans le cadre d'une recherche sur les films tournés par Roberto Rossellini avec Ingrid Bergman, en vue de leur restauration par le Centro Sperimentale di Cinematografia/Cineteca Nazionale (Rome). Rossellini a été commissaire extraordinaire du Centro Sperimentale di Cinematografia entre 1968 et 1969, et son président entre 1969 et 1974.

2. Sur ces trois versions et sur d'autres moins connues, $c f$. le chapitre dédié à ce film dans la recherche (à paraître) mentionnée à la note 1 .

3. Comme plus tard Angst/La paura (tourné deux fois, en allemand et en anglais) et Ingrid Bergman (épisode du film Siamo donne, dont une partie a été tournée en anglais et en italien), bien que cette fois-ci presque toutes les prises de vues de Stromboli aient été tournées en anglais. Pour une description détaillée de ces variantes, cf. la recherche (à paraître) citée à la note 1 .

4. Il n'est pas exclu que Rossellini ait utilisé les prises de vues RKO comme "preuves» filmées, à en juger par leur qualité, souvent inférieure.

5. Cf. entre autres Macorini 1987; cf. également la riche bibliographie publiée dans Anile et Giannice 2000. 
6. Cf. Anile et Giannice 2000 .

7. Cf. Rossellini 1968 (p. 212-213).

8. Voici ce qu'on peut lire dans la lettre: «I am so afraid that somebody MUST show how smart he is and start recutting."

9. Cf. Corriere dell'informazione, 26 janvier 1950.

10. Cf. Bergman et Burges 1980.

11. Le scénario fut réécrit plusieurs fois; au fur et à mesure, il est évident que se dessinent ces deux noyaux thématiques, qui seront traités différemment par Rossellini et par la RKO.

12. Cf. Aprà et Spagnoletti 1998.

13. $C f$. Brenez, «Montage intertextuel et formes contemporaines du remploi dans le cinéma expérimental », ailleurs dans le présent numéro de Cinémas.

14. Cf. Dagrada, Gaudreault et Gunning 1999 et Dagrada, Gaudreault et Gunning 2000 .

15. Sur la différence entre syntagme alterné et syntagme parallèle, $c f$. Metz 1968 (p. 127-130). Ici, il n'est jamais question d'alternance entre deux ou plusieurs séries dépourvues de liens spatio-temporels; bien au contraire, l'analyse de l'espace et son morcellement restent fondamentaux. Mais la mise en rapport qui s'instaure est d'ordre métaphorique et symbolique.

16. Ainsi peut-on lire dans le document original: «il misterioso personaggio è svelato sin dall'inizio sin nelle budella!» $C f$. ce document dans la recherche (à paraître) mentionnée à la note 1 .

\section{RÉFÉRENCES BIBLIOGRAPHIQUES}

Anile et Giannice 2000: Alberto Anile et Maria Gabriella Giannice, La guerra dei vulcani, Genève, Le Mani, 2000.

Aprà et Spagnoletti 1998: Adriano Aprà et Giovanni Spagnoletti (dir.), «Dossier Rossellini ", Close-Up, n 3, 1998.

Bergman et Burges 1980 : Ingrid Bergman et Alan Burges, Ingrid Bergman. My Story, New York, Delacorte Press, 1980.

Dagrada, Gaudreault et Gunning 1999: Elena Dagrada, André Gaudreault et Tom Gunning, "Composition en profondeur, mobilité et montage dans Cabiria (Pastrone, 1914)», Cinémas, vol. 10, no 1, 1999, p. 56-78.

Dagrada, Gaudreault et Gunning 2000 : Elena Dagrada, André Gaudreault et Tom Gunning, "Regard oblique, bifurcation et ricochet, ou de l'inquiétante étrangeté du carrello", Cinémas, vol. 10, ns 2-3, 2000, p. 207-223.

Macorini 1987 : Edgardo Macorini, «Sono la prova vivente della brutalità di Hollywood" [1950], sous le titre "Il caso Stromboli», dans Adriano Aprà (dir.), Il mio metodo, Venise, Marsilio, 1987.

Metz 1968 : Christian Metz, Essais sur la signification au cinéma, tome I, Paris, Klincksieck, 1968.

Rossellini 1968 : Renzo Rossellini, Addio del passato. Racconti ed altro, Milan, Rizzoli, 1968.

Hollywood, Rossellini et le syndrome de Griffith 